\title{
Looking to the future of cancer immunotherapy: many questions to answer and many therapeutic opportunities
}

\author{
Suzanne Ostrand-Rosenberg
}

Received: 24 November 2012/Accepted: 28 November 2012/Published online: 20 December 2012

(C) Springer-Verlag Berlin Heidelberg 2012

These are exciting times in the field of tumor immunology. Recent and ongoing immunotherapy clinical trials have validated the long-held belief that the immune system can be harnessed for the treatment of advanced cancer. Both antibody-mediated and immune cell-mediated strategies that enhance antitumor immunity have yielded encouraging and in some cases clinically positive responses that markedly increase the survival times of cancer patients. When Cancer Immunology, Immunotherapy (CII) was established in 1976, immunological intervention as a therapeutic modality for cancer was only a concept. CII provided one of the few venues exclusively focused on this evolving field. As the field of tumor immunology has matured and entered the mainstream of cancer therapy, so has CII, which continues to play a critical role by publishing important advances in the field. As I assume the position of Co-Editor-in-Chief, I am indebted to my predecessor, Henry Mihich, who devoted 32 years as Co-Editor-inChief and who through his leadership enabled CII to become an even more important platform for the presentation and discussion of data, hypotheses, and novel ideas.

At the same time promising immunotherapies are being developed, immune suppressive mechanisms that deter antitumor immunity and promote tumor escape are being discovered. Immunotherapies that target some of these suppressive mechanisms are demonstrating that immune suppression can be overcome; however, other suppressive mechanisms remain as significant obstacles to immunotherapy. Recent progress has also raised questions about how immunotherapy can be integrated with existing non-

S. Ostrand-Rosenberg $(\bowtie)$

Baltimore, MD, USA

e-mail: srosenbe@umbc.edu immunological therapies, as well as the feasibility of delivering immunotherapy to large numbers of patients.

In order to move the field forward, there are many strategic questions that must be addressed. For example: How can immunotherapy be best combined with conventional therapy for optimal synergy? Does combining independent immunotherapy approaches improve overall responses? Are antibody and cell-mediated immunotherapies additive or synergistic in their effects? Are immune suppressive mechanisms consistent between patients with the same type of cancer? Do immune suppressive mechanisms vary with stage of disease? Are there potent and widespread immune suppressive mechanisms that are still unknown? Do epigenetic alterations contribute to immune suppression or induce an ever-changing and evolving immune response? Will active immunotherapy be sufficiently long-lasting to protect individuals against outgrowth of quiescent metastatic cells? Can immunotherapy be combined with targeted oncogene therapy to improve overall responses? As tumors evolve in vivo, will they require different immunotherapy strategies? Will targeting cancer stem cells with immunotherapy be feasible and effective? Are there immunological biomarkers that reliably predict therapeutic outcomes? Can we identify better immunomonitoring indicators to predict patient outcome? Can methodologies be developed so that customized immunotherapies can be economically delivered to the general population? Some of these questions must first be addressed in preclinical systems. Such studies will require experiments with animals and necessitate developing more realistic animal models. Other questions may be initially tackled via translational or clinical studies. Regardless of which experimental approach is initially used, it is likely that definitive answers will only be obtained by comprehensive clinical studies combined with studies that provide 
a solid understanding of the basic mechanisms that induce and regulate immune responses in individuals with cancer.

Cancer Immunology, Immunotherapy will continue to play a key role in the field by publishing papers that report cutting edge mechanistic and clinical tumor immunology studies. Therefore, the journal welcomes the submission of manuscripts that address the many important issues in cancer immunology and immunotherapy that must be solved, so immunotherapy can become an efficacious mainstream treatment for cancer.

Suzanne Ostrand-Rosenberg

Co-Editor-in-Chief

Conflict of interest The author has no financial or commercial conflict of interest. 